\title{
A Hybrid Approach to Parallel Pattern Discovery in C++
}

\author{
C. Brown, V. Janjic, A. Barwell, J. Thomson \\ School of Computer Science \\ University of St Andrews \\ St Andrews, UK \\ \{vj32, cmb21, adb23,j.thomson\}@st-andrews.ac.uk \\ J.D. Garcia-Sanchez, D. Del Rio Astorga \\ Computer Science and Engineering Dept. \\ Universidad Carlos III de Madrid \\ Madrid, Spain \\ \{josedaniel.gracia, david.rio\}@uc3m.es
}

\author{
R. Castañeda Lozano, M. Cole, B. Franke \\ School of Informatics \\ University of Edinburgh \\ Edinburgh, UK \\ $\{$ rcastae, mic, bfranke\}@inf.ed.ac.uk \\ K. MacKenzie
IOHK \\ kenneth.mackenzie@iohk.io
}

\begin{abstract}
Parallel pattern libraries offer a strong combination of abstraction and performance. However, discovering places in sequential code where parallel patterns should be introduced is still highly non-trivial, often requiring expert manual analysis and profiling. We present a hybrid discovery technique to detect instances of parallel patterns in sequential code. This employs both static and dynamic trace-based analysis, together with hotspot detection. We evaluate our pattern discovery mechanism on a number of representative benchmarks. We evaluate the performance of the resulting parallelised benchmarks on a 24core parallel machine.
\end{abstract}

\section{INTRODUCTION AND BACKGROUND}

With the massive increase of scale of parallelism and heterogeneity of modern parallel hardware, programming parallel systems has now become harder than ever. Models based on parallel patterns have gained lots of traction recently [1, 2, 4], with significant industry players releasing their own pattern libraries. Pattern libraries offer functions for common parallel structures such as pipeline, map and reduce, which can be instantiated into appropriate places in otherwise sequential application code. However, exactly where in the code to introduce patterns and what pattern to introduce is challenging. In this paper we present a novel hybrid composition of both static and dynamic analyses of sequential applications to detect places where parallel patterns should be introduced. Our study involves three complementary components: profilingbased hotspot detection, static pattern detection on hotspot loops, and trace-based pattern detection on hotspot loops. In this way, we are able to answer both the where should parallelism be introduced and what type of parallelism should be introduced questions for an existing sequential application. We evaluate our approach and demonstrate that we are able to identify, among numerous loops in the benchmarks, those that are both computationally intensive and conform to one of the standard parallel patterns. In this paper, we restrict ourselves to two classical parallel patterns, which we believe to be the most widely known:

- The map pattern models a data parallel computation, where a single computational worker $f$ is applied to a set of independent inputs $x_{1}, \ldots, x_{n}$. Parallelism arises from applying the worker $f$ to input elements in parallel.

- The reduce pattern models a reduction operation, where a binary associative function $\oplus$ is applied pairwise to the elements of a set of inputs, $x_{1}, \ldots, x_{n}$. The output of the reduce becomes, $x_{1} \oplus x_{2} \oplus x_{3} \cdots \oplus x_{n}$. Parallelism arises by staging the reductions in parallel.

\section{Detecting Parallelism Candidates in C++}

Our parallelism discovery methodology is shown in Figure 1. Here, the programmer starts with a program written in C++. The hotspot analysis over the sequential code duplicates the original sequential program, instruments it with profiling code, executes the instrumented version, and records the results for use by the tracing stage. For each hotspot loop candidate, we apply two contrasting pattern-detection mechanisms to determine whether the loop is an instance of a parallel pattern. In static pattern-detection, each parallelism candidate hotspot is passed through a static analysis, leveraging the existing Pattern Analyzer Tool PPAT [3]. PPAT takes advantage of the clang lib-tooling utilities to statically analyze the code at the Abstract Syntax Tree level. It traverses the AST and collects information about the data dependencies, analyses them and introduces annotation indicating the potential parallel patterns in the form of $\mathrm{C}++$ attributes.

Independently from the static analysis, candidate hotspots are also passed through our new dynamic trace-based pattern detection mechanism (described in Section III). Finally, the user checks manually that the candidate detected patterns (from either analysis) are indeed applicable. A detected pattern might not be applicable for different reasons, for example if 


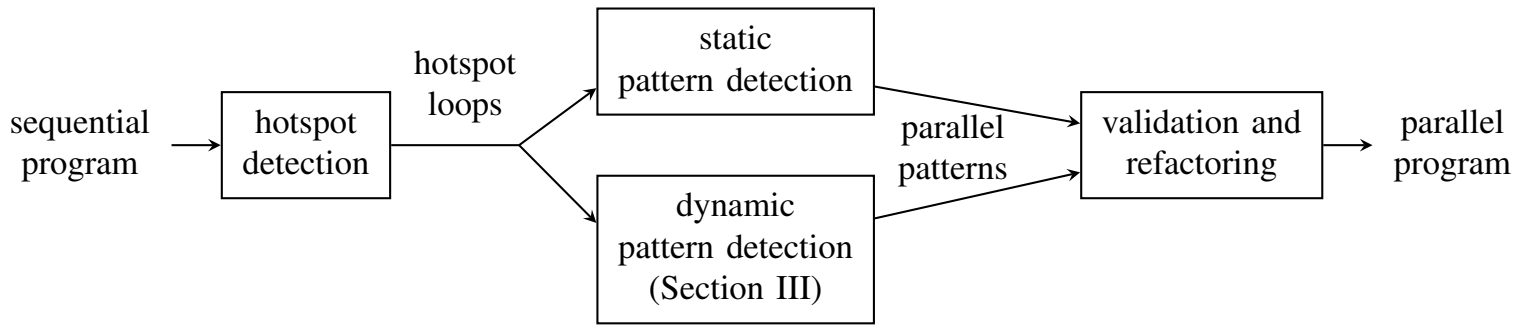

Fig. 1: Overview of our approach to parallel pattern discovery.

the input used for tracing does not uncover all dependencies across loop iterations.

As the fork in the diagram suggests (see Figure 1), in our current prototype we apply both static and dynamic approaches to all candidate loops. This recognizes that each technique could find instances of potential patterns undetected by the other. It also allows us to compare the performance of the two techniques directly. In a production system, we could decide to only apply the more computationally expensive dynamic method to candidates for which the static approach has not found an acceptable pattern.

\section{Dynamic Trace-Based Detection of Patterns IN HOT SPOTS}

Our new dynamic trace-and-match method detects whether a hotspot loop corresponds to a parallel pattern. The method proceeds in two steps: first, it constructs a graph representation of the execution of the loop (Section III-A) by instrumenting and tracing the loop's data flow. Then, it applies pattern matching techniques to check whether the loop iterations correspond to a parallel pattern (Section III-B).

This paper explores the matching of map and reduce patterns. Generalizing the method to match more complex patterns such as pipeline and stencil is part of ongoing work.

\section{A. Dynamic Dependency Graphs}

The pattern detection method represents input loops as Dynamic Dependency Graphs (DDG). A DDG is a directed acyclic graph in which nodes correspond to executions of instructions (or groups of instructions), and arcs correspond to flow of data across them. The DDG is a natural representation of the essential computation of a program, as it abstracts away implementation aspects such as the choice of data structures or order of computation [9].

Our method constructs the DDG of a loop by instrumenting the loop at the compiler intermediate representation (IR) level and tracing its data flow as the loop is executed.

The size of DDGs grows quickly with the number of executed instructions and can make pattern matching prohibitive. To reduce this size, sets of nodes corresponding to loop body instructions from the same iteration are merged into single nodes. This simplification leads to DDGs containing two types of nodes: loop body nodes and single instruction nodes corresponding to iterator instructions such as induction variable increments.

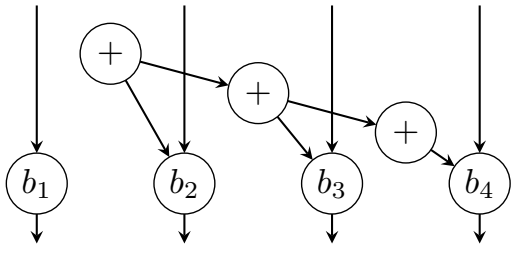

Fig. 2: DDG of a map style for loop iterated four times. Each $b_{i}$ node corresponds to all loop body instructions in iteration $i$.

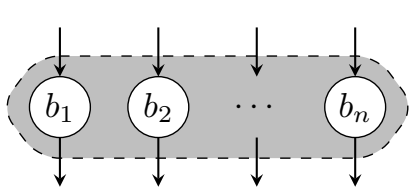

(a) Map pattern shape.

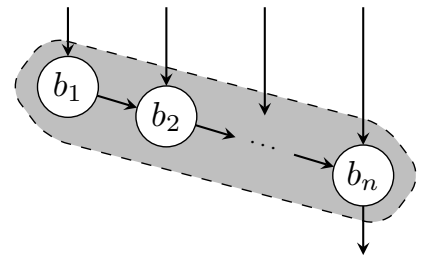

(b) Reduce pattern shape.
Fig. 3: Pattern shapes on DDGs.

Figure 2 shows an example DDG corresponding to an execution of four iterations of a typical map style for loop, where an induction variable is incremented in each iteration. The DDG includes addition nodes $(+)$ that correspond to the increment of the induction variable and loop body nodes $\left(b_{i}\right)$ that correspond to instructions executed in iteration $i$.

\section{B. Matching of Parallel Patterns}

The pattern detection method checks, for the simplified loop DDG, whether the loop body nodes match the shape of a map or reduce pattern. This check is formulated and performed as a graph pattern matching problem, where pattern matches are found by testing the satisfiability of pattern-specific constraints over the set of loop body nodes.

Figure 3 depicts the shape of map and reduce patterns on DDGs containing a set of loop body nodes $B=$ $\left\{b_{1}, b_{2}, \cdots, b_{n}\right\}$. Such a set matches a map pattern if and only if its nodes are disconnected:

$$
b_{j} \text { is not reachable from } b_{i} \quad \forall b_{i}, b_{j} \in B: b_{i} \neq b_{j} .
$$

For example, the body nodes $\left\{b_{1}, b_{2}, b_{3}, b_{4}\right\}$ in Figure 2 match a map pattern, as none of them reach each other in the DDG. 
A set of loop body nodes $B$ matches a reduce pattern if and only if its nodes form a path where the outdegree of all but the last node is equal to one:

$$
\operatorname{successors}\left(b_{i}\right)=\left\{b_{i+1}\right\} \quad \forall i: 1 \leq i<n .
$$

These pattern definitions are solely based on data-flow structure, and do not take into account other criteria such as specific relations among different input data elements. This high-level, structural view simplifies and broadens the scope of pattern detection, for example allowing the detection of potential patterns across scattered data elements or different data collections. However, it also implies that our method can potentially propose invalid pattern matches that do not satisfy additional criteria besides matching the pattern's dataflow structure. Examples are pattern matches containing noncommutative system calls and reduce matches where the loop body is not an associative reduction operator. Reducing the number of invalid pattern matches is part of future work.

\section{REsults}

\section{A. Parallel Pattern Detection Performance}

This section studies the pattern detection capabilities of the static PPAT tool and the dynamic trace-and-match method described in Section IIII and identifies strengths, limitations, and opportunities based on the results.

Table I compares the map and reduce patterns detected by both approaches with those detected by a manual expert analysis for each of the loops selected by hotspot analysis. For the dynamic approach, we have traced each benchmark using small input data sets, to reduce the size of the resulting dependency graphs while executing each of the targeted loops a sufficient number of iterations.

Table III summarizes the performance of each pattern detection method using the expert analysis results as the ground truth. The performance of each method is summarized by three measures: accuracy (percentage of loops classified correctly, whether containing patterns or not), precision (percentage of actual patterns among all detected patterns), and recall (percentage of detected patterns among all actual patterns).

The results show that, across the selected benchmarks, both pattern detection methods have a substantial overall accuracy $(79 \%)$. As can be expected from their fundamental properties, the methods present complementary strengths: while the static pattern detector is sound ( $100 \%$ precision), the dynamic pattern detector trades some precision $(-18 \%)$ for higher overall recall $(+15 \%)$.

The precision results confirm the soundness of the static pattern detector, and show that the dynamic pattern detector has a lower yet substantial overall precision (82\%), only affected by four loops where patterns are falsely detected. Loop 134 (Ant Colony) is a max reduction implemented with a conditional assignment. The dynamic detector abstracts away assignments that move but do not transform data, and falsely detects the resulting instructions as a map pattern. Loop 373 (Black-Scholes) contains non-commutative system

\begin{tabular}{|c|c|c|c|c|c|c|c|c|}
\hline \multirow{2}{*}{ benchmark } & \multirow{3}{*}{$\begin{array}{c}\begin{array}{c}\text { loop } \\
\text { ID }\end{array} \\
258\end{array}$} & \multirow{2}{*}{ exec. } & \multicolumn{3}{|c|}{ map pattern } & \multicolumn{3}{|c|}{ reduce pattern } \\
\hline & & & static & dynam & expert & static & dynam. & expert \\
\hline \multirow{8}{*}{ Ant Colony } & & $99 \%$ & 0 & 0 & 0 & 0 & 0 & 0 \\
\hline & 259 & $84 \%$ & 0 & - & - & 0 & 0 & 0 \\
\hline & 120 & $84 \%$ & 0 & 0 & 0 & 0 & 0 & 0 \\
\hline & 123 & $51 \%$ & 0 & $\bigcirc$ & $\bullet$ & 0 & 0 & 0 \\
\hline & 211 & $15 \%$ & - & - & - & 0 & 0 & 0 \\
\hline & 90 & $14 \%$ & - & - & - & 0 & 0 & 0 \\
\hline & 212 & $12 \%$ & - & - & - & 0 & 0 & 0 \\
\hline & 134 & $8 \%$ & 0 & 0 & 0 & 0 & 0 & 0 \\
\hline \multirow{3}{*}{ Black-Scholes } & 285 & $40 \%$ & 0 & 0 & $\bullet$ & 0 & 0 & 0 \\
\hline & 290 & $24 \%$ & 0 & 0 & 0 & 0 & 0 & 0 \\
\hline & 373 & $3 \%$ & 0 & - & 0 & 0 & 0 & 0 \\
\hline \multirow{5}{*}{ Convolution } & 158 & $100 \%$ & 0 & - & 0 & O & 0 & O \\
\hline & 112 & $99 \%$ & $\bullet$ & ○ & $\bullet$ & 0 & 0 & 0 \\
\hline & 111 & $99 \%$ & $\bullet$ & 0 & 0 & 0 & 0 & 0 \\
\hline & 119 & $94 \%$ & 0 & 0 & 0 & 0 & 0 & 0 \\
\hline & 120 & $45 \%$ & 0 & 0 & 0 & 0 & 0 & 0 \\
\hline \multirow{3}{*}{ Mandelbrot } & 60 & $96 \%$ & - & - & - & 0 & 0 & 0 \\
\hline & 62 & $96 \%$ & $\bullet$ & 0 & 0 & 0 & 0 & 0 \\
\hline & 33 & $90 \%$ & 0 & 0 & 0 & 0 & 0 & 0 \\
\hline \multirow{10}{*}{ Transfil } & 54 & $99 \%$ & 0 & 0 & 0 & 0 & 0 & 0 \\
\hline & 233 & $90 \%$ & 0 & 0 & 0 & 0 & 0 & 0 \\
\hline & 78 & $60 \%$ & 0 & 0 & 0 & 0 & 0 & 0 \\
\hline & 94 & $60 \%$ & 0 & 0 & 0 & 0 & 0 & 0 \\
\hline & 157 & $59 \%$ & 0 & 0 & 0 & 0 & 0 & 0 \\
\hline & 183 & $45 \%$ & 0 & - & ? & 0 & 0 & 0 \\
\hline & 90 & $45 \%$ & 0 & 0 & 0 & 0 & 0 & 0 \\
\hline & 120 & $39 \%$ & 0 & 0 & 0 & 0 & 0 & 0 \\
\hline & 217 & $5 \%$ & 0 & 0 & 0 & 0 & 0 & 0 \\
\hline & 464 & $2 \%$ & 0 & 0 & 0 & 0 & 0 & 0 \\
\hline
\end{tabular}

TABLE I: Patterns detected by the static and dynamic approaches and by expert analysis in the loops selected by hotspot analysis. The loops within each benchmark are sorted in descending order of execution time (exec.). Filled circles (O) indicate that the corresponding pattern is detected, empty circles $(\bigcirc)$ indicate the opposite.

\begin{tabular}{lcccccc}
\hline \multirow{2}{*}{ pattern } & \multicolumn{2}{c}{ accuracy } & \multicolumn{2}{c}{ precision } & \multicolumn{2}{c}{ recall } \\
& static & dynamic & static & dynamic & static & dynamic \\
\hline map & $62 \%$ & $72 \%$ & $100 \%$ & $88 \%$ & $48 \%$ & $71 \%$ \\
reduce & $97 \%$ & $86 \%$ & $100 \%$ & $60 \%$ & $80 \%$ & $60 \%$ \\
\hline total & $79 \%$ & $79 \%$ & $100 \%$ & $82 \%$ & $54 \%$ & $69 \%$ \\
\hline
\end{tabular}

TABLE II: Pattern detection performance.

calls (fscanf) which preclude parallelization in practice. Loops 258 (Ant Colony) and 120 (Transfil) are structured as reduce patterns but their body is not associative.

The static pattern detector misses a total of twelve map and reduce patterns, due to its conservativeness in the face of potential side-effects, pointer aliasing, and unknown information at compile time. The dynamic pattern detector misses eight actual patterns. In Loops 123 (Ant Colony), 119 and 120 (Convolution), and 217 (Transfil), the Dynamic Dependency Graph simplification described in Section III-A precludes detecting partial patterns that only cover some of the instructions in the loop body. In Loop 134 (Ant Colony), the dynamic approach misses the reduce pattern due to the assignment instruction limitation described above. Finally, in 
Loops 54 and 78 (Transfil), the dynamic approach fails to find map patterns due to loop-carried dependencies induced by output buffering, pseudo-random number generation, and intermediate result caching. While these dependencies could be in principle removed by a manual expert, the transformations required are beyond the scope of our detection methods.

Stencil pattern case study. Regarding the Convolution benchmark using the static pattern analyzer, additionally to the map patterns detected by the dynamic and static approaches, it also detects an instance of a stencil pattern in Loop 112. Listing 1 shows an excerpt of the annotated loop by the tool. As observed, the static analyzer detected the loop as a stencil pattern since each output item can be transformed independently and depend on multiple input data items. Furthermore, the tool also annotated the input/output parameters of the pattern in order to ease the code transformation by a developer.

Listing 1: Annotated stencil pattern in Convolution benchmark

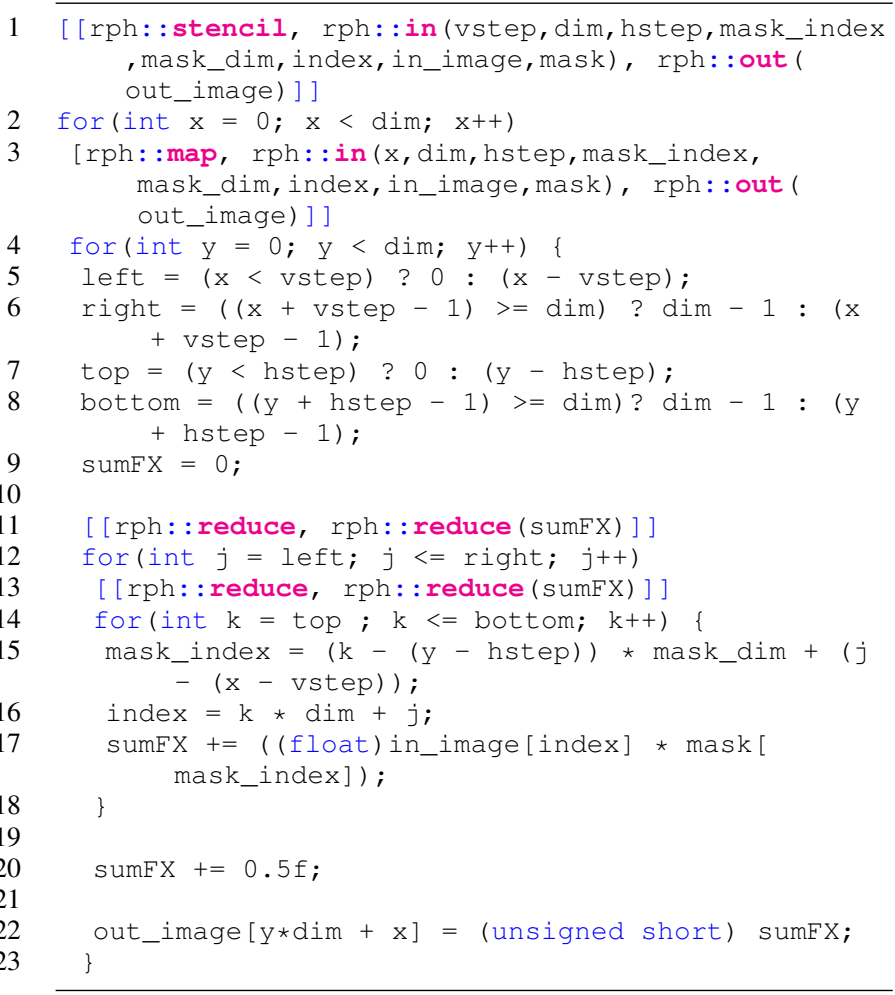

\section{B. Parallel Pattern Runtime Performance}

For each benchmark, we have taken the loop reported as having the highest execution time by the hotspot analysis and also reported as a map pattern by at least one of the pattern detectors. Our goal is to verify whether the execution time of the identified loops translates into highly effective speedups.

All of our execution experiments are conducted on a server with Intel Xeon E5-2690 CPU with 28 cores, running at 2.6 GHz with 256 GB of RAM, with Scientific Linux 6.2.

The performance results for the Mandelbrot, Convolution, Ant Colony, and Black-Scholes benchmarks are shown in

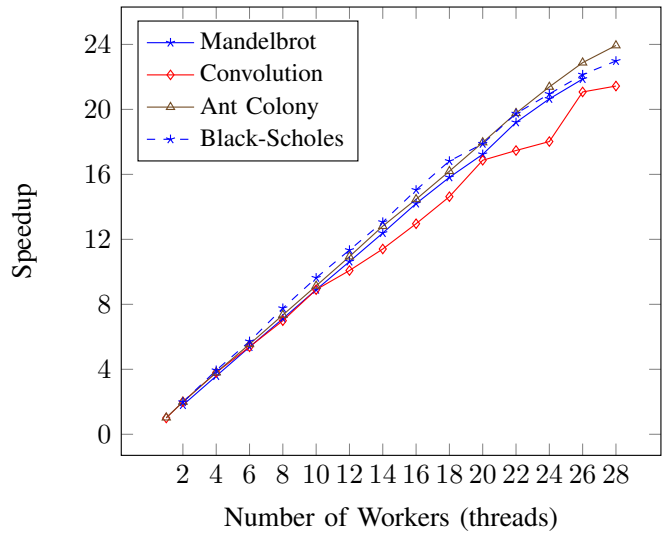

Fig. 4: Speedup results for Mandelbrot, Convolution, Ant Colony, and Black-Scholes where the hottest map pattern is parallelized.

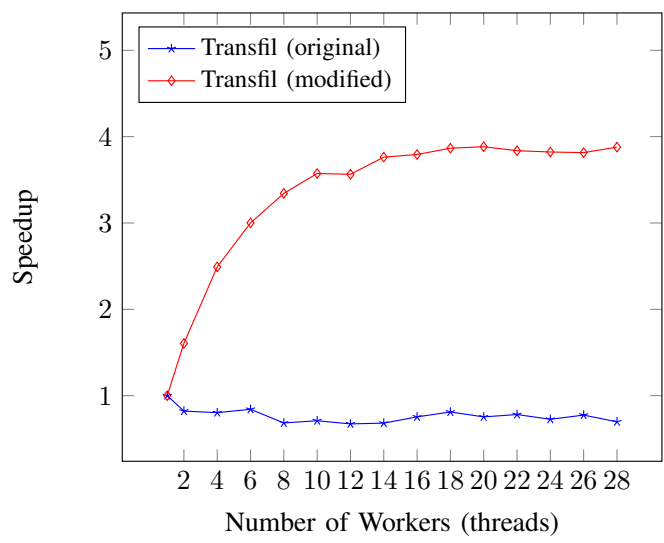

Fig. 5: Speedup results for Transfil (original and modified) where the hottest map pattern (Loop 233) is parallelized.

Figure 4. These benchmarks show significant speedups, with Mandelbrot (Loop 60), Ant Colony (Loop 259) and BlackScholes (Loop 285) all reaching speedups of around 22 on 28 cores. Convolution starts to plateau at around 18 cores, with a speedup of 16 , but steadily increases up to a speedup of around 21 on 28 cores.

The performance results for the Transfil benchmark (where Loop 233 is parallelized) are shown in Figure 5 (Transfil original). As can be seen, Transfil differs radically from the other benchmarks in that it does not shown any speedup, even though Loop 233 takes $90 \%$ of its total execution time. The key bottleneck in this loop is the intensive use of a single random number generator that imposes a serialization among the different threads accessing it. Detecting such performance bottlenecks is currently outside the scope of our discovery technique.

The effect of using a single random number generator can be noticed by comparing the speedup of the original Transfil benchmark (Transfil original in Figure 5) with the speedup of a modified version (Transfil modified in the same figure) where the most frequent random generation calls are replaced 
by numeric constants. In the latter case, reasonable speedup is obtained, suggesting that each thread should use its own random number generator to obtain an effective parallelization.

\section{RELATED WORK}

There is a significant amount of previous work on detecting parallelism by tracing data dependencies dynamically [5, 6, 7, 8, 10, 11]). Among these approaches, those by Rul et al. [10] and Huda et al. [6] are most closely related to our trace-and-match method from Section III. Both approaches aim at detecting map and pipeline patterns (the latter also targets reduce patterns) on different program representations based on profiled data- and control-flow information. Both Rul et al. [10] and Huda et al. [6] use graphs as the main abstraction in their representations, but, unlike our approach, they aggregate data and control-flow information about different executions of the same program region into single nodes. While this yields a more compact representation, we believe that conserving specific information about different executions of a program region is needed to detect patterns that act differently depending on input data and other dynamic properties, which we aim to explore in future work. Rul et al. [10] track dependencies at the data structure level, while Huda et al. [6] use read-compute-write units. In contrast, our method admits varying levels of granularity based on static program structure. We plan to explore adapting the granularity during pattern detection to balance the trade-off between scalability and detection performance. As discussed in Section IV-A. a finer granularity would increase significantly the scope of detectable patterns.

\section{CONCLUSIONS AND Future WORK}

In this paper, we presented a new hybrid-approach for parallel pattern discovery in $\mathrm{C}++$. This novel discovery approach employs three kinds of analysis to find instances of parallel patterns, including dynamic hotspot detection that profiles the execution with monotonic runtime timing, static analysis using the PPAT tool, and dynamic trace-based methods, which analyse the execution data flow to determine instances of patterns. Driven by candidates suggested by hotspot analysis, both pattern-detection techniques reported instances of parallel patterns in each of the benchmarks reported. We confirmed that instances in the source-code indeed could be transformed into instances of parallel patterns with significant speedups of up to 22 on a 28 -core machine. In the future, we plan to extend our hybrid pattern discovery technique further, in order to support additional patterns, such as divide and conquer and to other languages as well. We also plan to evaluate our hybrid discovery technique on larger use-cases. Finally, we intend to refine the technique to take into account different types of scalability bottlenecks.

\section{ACKNOWLEDGEMENTS}

This work was supported by the EU Horizon 2020 project, TeamPlay, grant number 779882, and UK EPSRC Discovery, grant number EP/P020631/1.

\section{REFERENCES}

[1] Marco Aldinucci, Marco Danelutto, Peter Kilpatrick, and Massimo Torquati. Fastflow: High-Level and Efficient Streaming on Multicore, chapter 13, pages 261-280. John Wiley \& Sons, Ltd, 2017.

[2] Jeffrey Dean and Sanjay Ghemawat. MapReduce: Simplified data processing on large clusters. In Proceedings of the 6th Conference on Symposium on Operating Systems Design \& Implementation - Volume 6, OSDI'04, pages 10-10, Berkeley, CA, USA, 2004. USENIX Association.

[3] David del Rio Astorga, Manuel F Dolz, Luis Miguel Sánchez, J Daniel García, Marco Danelutto, and Massimo Torquati. Finding parallel patterns through static analysis in $\mathrm{C}++$ applications. The International Journal of High Performance Computing Applications, 32(6): 779-788, 2018.

[4] Johan Enmyren and Christoph W. Kessler. SkePU: A multi-backend skeleton programming library for multiGPU systems. In Proceedings of the Fourth International Workshop on High-level Parallel Programming and Applications, HLPP '10, pages 5-14, New York, NY, USA, 2010. ACM.

[5] Fabian Gruber, Manuel Selva, Diogo Sampaio, Christophe Guillon, Antoine Moynault, Louis-Noël Pouchet, and Fabrice Rastello. Data-flow/dependence profiling for structured transformations. In Principles and Practice of Parallel Programming, pages 173-185, New York, NY, USA, 2019. ACM.

[6] Zia Ul Huda, Rohit Atre, Ali Jannesari, and Felix Wolf. Automatic parallel pattern detection in the algorithm structure design space. In International Parallel and Distributed Processing Symposium, pages 43-52, Washington, DC, USA, May 2016. IEEE.

[7] Alain Ketterlin and Philippe Clauss. Profiling datadependence to assist parallelization: Framework, scope, and optimization. In IEEE/ACM International Symposium on Microarchitecture, MICRO-45, pages 437-448, Washington, DC, USA, 2012. IEEE.

[8] Zhen Li, Ali Jannesari, and Felix Wolf. Discovery of potential parallelism in sequential programs. In Parallel Processing, pages 1004-1013, Washington, DC, USA, 2013. IEEE.

[9] Nicholas Nethercote and Alan Mycroft. Redux: A dynamic dataflow tracer. Electronic Notes in Theoretical Computer Science, 89(2), 2003.

[10] Sean Rul, Hans Vandierendonck, and Koen De Bosschere. A profile-based tool for finding pipeline parallelism in sequential programs. Parallel Computing, 36 (9):531-551, September 2010.

[11] Georgios Tournavitis and Björn Franke. Semi-automatic extraction and exploitation of hierarchical pipeline parallelism using profiling information. In Parallel Architectures and Compilation Techniques, PACT '10, pages 377-388, New York, NY, USA, 2010. ACM. 